\title{
Strategies for Multimodal Analysis of Joint EELS and EDS Data
}

Ray Twesten ${ }^{1}$, Jens Rafaelsen ${ }^{2}$, Liam Spillane ${ }^{3}$ and Paul Thomas ${ }^{3}$

${ }^{1}$ Gatan Inc., United States, ${ }^{2}$ Ametek / EDAX LLC, Mahwah, New Jersey, United States, ${ }^{3}$ Gatan Inc., Pleasanton, California, United States

The complementary nature of electron energy-loss spectroscopy (EELS) and energy dispersive [x-ray] spectroscopy (EDS) signals makes it highly desirable to acquire both during electron microscopy investigations of materials. With ongoing improvements in instrumentation and detectors, it is now common to acquire joint EELS-EDS spectrum image data for materials analysis, all the way from large area mapping down to atomic scale analysis. Having all data types on a single platform opens new avenues for data analysis and interpretation.

In the table in Figure 1, we show several of the complementary aspects of the excitation (EELS) and deexcitation (EDS) process; by exploiting the strengths of each, a more complete analysis is possible. For example, in thin specimens, the characteristic x-rays sit atop a relatively low background giving a high peak-to-background ratio. This allows long acquisitions from large volumes of material to detect and identify trace elements in the sample. However, due to scattering and secondary excitations, it is impossible to separate system peaks and non-local x-rays from true signals. The highly localized excitation process allows the EELS signal to confirm and verify the trace elements even for the most troubling system peaks.

Low-loss EELS contains a direct measure of the probe current and sample thickness in terms of the local mean free path. That information coupled with measured sample composition from both the core-loss EELS and the EDS signal allows the absolute sample thickness to be estimated [1, 2]. The measured thickness and an initial estimate of the local composition can be used to correct the signal for absorption [3] and generate a new estimate for the sample thickness in an iterative process.

Figure 2 shows the analysis of a joint EELS and EDS data set from an Fe-nitride precipitate in a GaN matrix. Low-loss EELS is used calculate the relative thickness and, combined with core-loss EELS and EDS, the absolute thickness is obtained. The local composition can be mapped using either EELS or EDS in this case. The composition in the bulk is close to $\mathrm{GaN}$ while the precipitate has over 55\% Fe. Overlap with the bulk cannot be ruled out; however, the N K-edge shows a clear ELNES change in the precipitate indicating compound formation. Using concurrent ELNES standards [4], the different chemical phases of nitrogen can be quantified. The defective region on the left of the precipitate shows a clear rise in the Fe L3 white line and nitrogen fine structure intermediate between the GaN bulk and the precipitate (Figure $2 \mathrm{~g}, \mathrm{~h}$ ). It is not clear what is causing this defect, but the magnetic properties may be affected.

In this presentation, we will discuss the software and hardware tools used to acquire and analyze these multimodal datasets to achieve an analysis greater than the sum of the individual techniques. 


\begin{tabular}{|c|c|c|}
\hline Property & Excitation (EELS) & De-excitation (EDS) \\
\hline \multirow{2}{*}{ Beam current } & Measure from ZLP & \\
\hline & Calibrate dose / dose-rate & Use current for quantification \\
\hline \multirow{2}{*}{ Absolute thickness } & Measure from low-loss; & \\
\hline & Correct quantification for diffraction & Correct quantification for absorption \\
\hline $\begin{array}{c}\text { Elemental } \\
\text { identification }\end{array}$ & $\begin{array}{l}\text { Exploit high localization to reject } \\
\text { artefact and system peaks }\end{array}$ & $\begin{array}{l}\text { Exploit high signal-to-background to } \\
\text { find trace element }\end{array}$ \\
\hline $\begin{array}{l}\text { Compositional } \\
\text { mapping }\end{array}$ & $\begin{array}{l}\text { Exploit high sensitivity to map thin } \\
\text { and dose sensitive regions }\end{array}$ & $\begin{array}{l}\text { Exploit high signal-to-background to } \\
\text { map thick regions }\end{array}$ \\
\hline \multirow{3}{*}{ Quantification } & $\begin{array}{l}\text { Well defined instrumentation factors } \\
\text { for light elements }\end{array}$ & $\begin{array}{l}\text { Large energy range in every } \\
\text { spectrum }\end{array}$ \\
\hline & $\begin{array}{c}\text { High sensitivity down to zero } \\
\text { thickness }\end{array}$ & Rare to miss an element \\
\hline & $\begin{array}{l}\text { Able to separate most overlapping } \\
\text { elements }\end{array}$ & $\begin{array}{l}\text { Often multiple lines to for each } \\
\text { element }\end{array}$ \\
\hline \multirow{3}{*}{$\begin{array}{l}\text { Chemical state / } \\
\text { physical properties }\end{array}$} & $\begin{array}{l}\text { Use ELNES to quantify chemical } \\
\text { bonding and oxidation state changes }\end{array}$ & \\
\hline & $\begin{array}{l}\text { Use plasmons to identify even subtle } \\
\text { changes in composition and density }\end{array}$ & \\
\hline & $\begin{array}{l}\text { Probe phonon and electronic band } \\
\text { structure with momentum resolved } \\
\text { EELS }\end{array}$ & \\
\hline
\end{tabular}

Figure 1. Complementary information provided by multimodal EELS and EDS data analysis. 


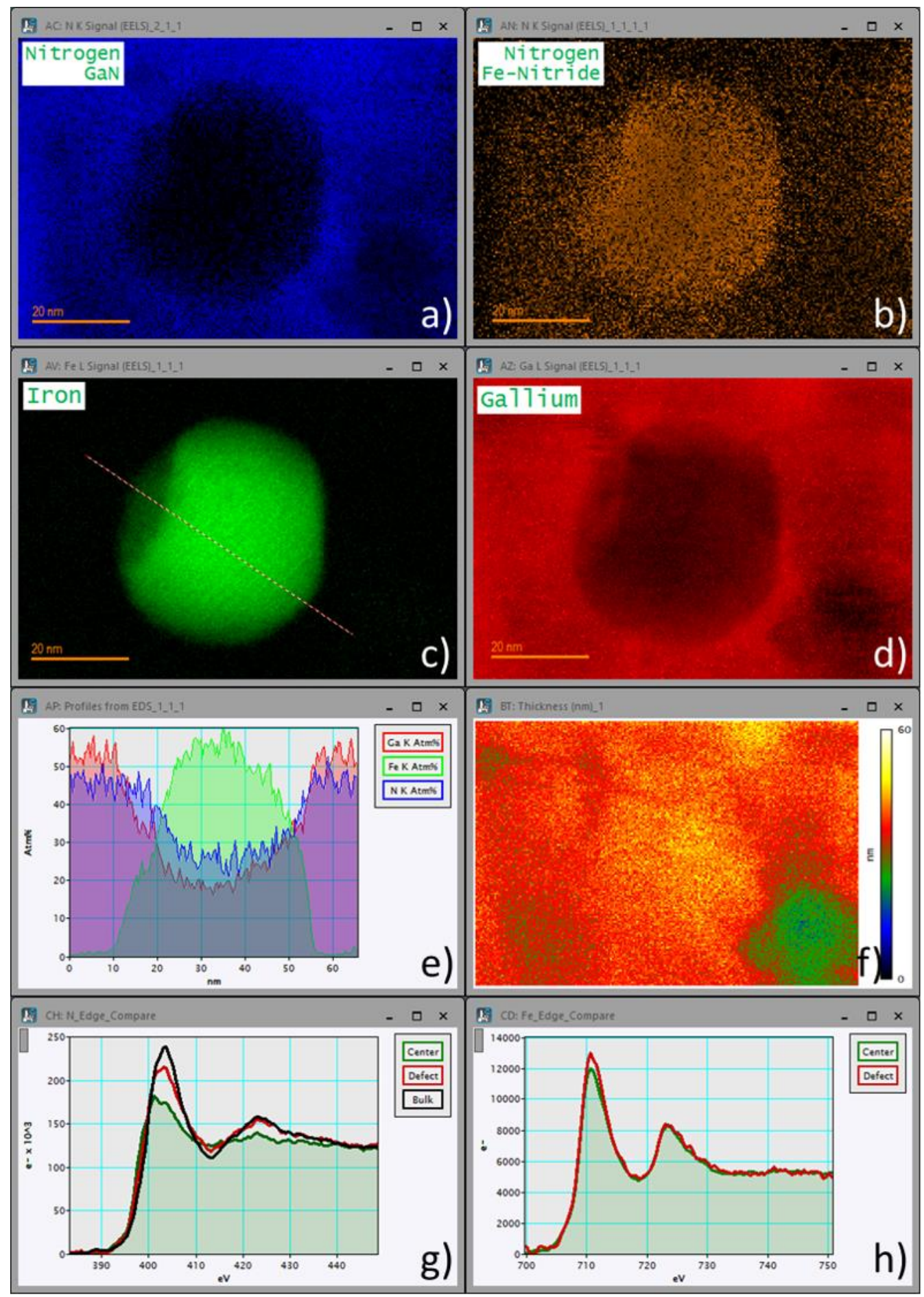

Figure 2. Joint analysis of an Fe-nitride precipitate in GaN. a) and b) chemical maps of N K edge using concurrent standards.; c) Fe L edge map; d) Ga L edge map; e) composition profiles (along the line in c) extracted from EDS data co-acquired with the EELS data; f) thickness map from EELS analysis. g) N-K edge fine structure from bulk and center of precipitate showing clear bonding changes in the nitrogen. The defective region on the left of the precipitate (dark green region in c) shows an intermediate nitrogen form, but it likely contains overlap between the GaN and the defect. h) The chemical change in the defect can 
be seen in the Fe L23 edge where there is slight dip in the L3 white line possibly indication a change in spin state.

References

[1] Iakoubovskii, K., Mitsuishi, K., Nakayama, Y., \& Furuya, K. (2008). Phys Rev B, 77(10), 104102.

[2] Malis, T., Cheng, S. C., \& Egerton, R. F. (1988). J. of Electron Microscopy Tech., 8(2), 193-200.

[3] Cliff, G., \& Lorimer, G. (1975). Journal of Microscopy, 103(2), 203-207.

[4] Schaffer, B., Spillane, L., \& Thomas, P. J. (2019). Microscopy and Microanalysis, 25(S2), 648-649. 